\title{
Enhanced Gene Delivery to Human Primary Endothelial Cells Using Tropism-Modified Adenovirus Vectors
}

\author{
Meredith A. Preuss ${ }^{*}, 1$, Joel N. Glasgow ${ }^{1,2,3}$, Maaike Everts ${ }^{1,2,4}$, Mariam A. Stoff-Khalili ${ }^{1}$, Hongju \\ $\mathrm{Wu}^{1,2}$ and David T. Curiel*,1,2
}

${ }^{1}$ Division of Human Gene Therapy, Departments of Medicine, Obstetrics and Gynecology, Pathology, Surgery and the
${ }^{2}$ Gene Therapy Center, ${ }^{3}$ Division of Cardiovascular Disease, ${ }^{4}$ Division of Molecular and Cellular Pathology, University
of Alabama at Birmingham, Birmingham, Alabama, 35294

Abstract: Endothelial cells have been noted to have relatively low expression of the native receptor for adenovirus serotype 5 (Ad5), coxsackie and adenovirus receptor (CAR), and are thus refractory to Ad5 infection. In this study, we hypothesize that increases in the infectivity of Ad5 in primary human pulmonary artery (HPAEC), coronary artery (HCAEC) and umbilical vein endothelial cells (HUVEC) can be achieved through genetic capsid modification of Ad5 to bypass CAR-dependent infection. The modifications tested in this study include incorporation of an integrin-binding RGD peptide motif (Ad5.RGD), a poly-lysine motif (Ad5.pK7), a combination of both of these peptide domains (Ad5.RGD.pK7), an adenovirus serotype 3 knob domain (Ad5/3Luc1) and canine adenovirus serotype 1 or 2 knob domains (Ad5Luc1-CK1 and Ad5Luc1-CK2). In HPAEC and HCAEC, the greatest infectivity enhancements were achieved using Ad5/3Luc1 (26fold and 30-fold respectively). HUVEC was most readily infected by Ad5Luc1-CK1 (213-fold). These results demonstrate that gains in Ad5 infectivity in endothelial cells can be accomplished with genetic capsid modifications.

\section{INTRODUCTION}

Endothelial cells play a vital role in regulation of blood flow to organs, immune cell trafficking and signaling, and are key regulators of the transport of oxygen and vital nutrients. The endothelium is now being recognized as a heterogeneous organ containing many different cell subsets with distinct locations and functions, and has been implicated as a key tissue in a growing number of human diseases [1]. On this basis, the ability to modulate gene expression in endothelial cells will play an important role in the study of basic endothelial biology and therapy development.

Vectors based on adenovirus serotype 5 (Ad5) have been a rational choice as gene delivery vehicles due to their well characterized biology, large transgene capacity and efficient gene transfer to dividing and non-dividing cell targets [2]. The first step in Ad5 infection occurs via high-affinity binding of the knob domain of the fiber protein to its cognate cellular receptor known as the coxsackie and adenovirus receptor (CAR) (Fig. 1) [3-6]. Ad5 is then internalized via receptor-mediated endocytosis by interaction of the penton base Arg-Gly-Asp (RGD) motif with cellular integrins $\alpha v \beta 3$, $\alpha v \beta 3, \alpha v \beta 1, \alpha 3 \beta 1$, or other integrin molecules [7-12]. In cellular endosomes, the Ad5 virion sheds some capsid proteins, escapes the endosomes and is subsequently transported to the nucleus where the Ad5 genes are then transcribed.

Since Ad5 infection is dependent upon the initial interaction with CAR, the presence of CAR on the host cell's

\footnotetext{
*Address correspondence to these authors at the University of Alabama at Birmingham, Biomedical Research Building II, $90119^{\text {th }}$ St S, Room 410, Birmingham, AL 35294, Tel: 205-975-0172; Fax: 205-975-8565; E-mail: mpreuss@uab.edu and University of Alabama at Birmingham, Biomedical Research Building II, $90119^{\text {th }}$ St S, Room 502, Birmingham, AL 35294; Tel: 205-934-9516; Fax: 205-975-7476; E-mail: curiel@uab.edu
}

surface is the crucial dictate of efficient infection. In this regard, non-target cells that express high levels of CAR can be infected, while target tissues, if low in CAR expression, are resistant to Ad5 infection [13]. Of note, endothelial cells have been observed to be relatively refractory to Ad5 infection and gene delivery due to low CAR expression $[14,15]$. Therefore, the development and employment of tropismmodified Ad5 vectors with CAR-independent cell entry pathways should provide efficient gene delivery to endothelial cells. Since the fiber protein, which contains the knob domain, is a major capsid determinant of Ad5 tropism, these tropism-modified vectors contain genetically modified fiber proteins. These modifications include the incorporation of small heterologous ligands as well as knob domains from alternate human Ad serotypes or non-human xenotypes that use CAR-independent cell attachment.

In this study, we evaluated tropism modified Ad5 vectors in three types of primary human endothelial cells that are commonly employed for in vitro study of vascular disease and endothelial cell biology. We hypothesized that these vectors, which have previously shown increased infectivity in other CAR-deficient cells [2], would demonstrate increased infectivity in primary human endothelial cells.

\section{MATERIALS AND METHODOLOGY}

\section{Tropism-Modified Adenoviral Vectors}

In this study, we evaluated Ad5 vectors with the following capsid modifications that have been previously described: incorporation of the integrin-binding RGD peptide (Ad5.RGD) and a poly-lysine (Ad5.pK7) motif into the HI loop of fiber [16, 17], human adenovirus serotype 3 knob domain (Ad5/3Luc1) [18], and canine adenovirus serotype 1 or 2 knob domains (Ad5Luc1-CK1 and Ad5Luc1-CK2) [19, 20]. The RGD and $\mathrm{pK} 7$ Ad5 vectors contain a dual reporter 
cassette that contains luciferase and green fluorescent protein. Ads that contain an alternate knob domain (Ad5/3Luc1, Ad5Luc1-CK1 and Ad5Luc1-CK2) contain a single luciferase reporter gene. All tropism-modified Ad5 vectors were compared to an Ad5 vector with wild type fiber that also contained an identical reporter gene expression cassette. All vectors in this study were replication defective due to the deletion of the $\mathrm{E} 1$ region of the Ad5 genome.

The determination of plaque forming units (PFU) is usually measured by the virus' ability to enter and replicate in HEK293 cells. These cells are CAR-positive, and are thus a good substrate for Ad5-based gene therapy vectors. The vectors described in this manuscript have a CAR-independent infectivity pathway. The target receptor for some of these vectors still remains to be identified, and may or may not be present on the surface of HEK293 cells. Determining PFU levels will therefore not necessarily correlate with the true infection and replication potential of the capsid-modified virions. We therefore decided to use VP numbers as the titer of the various preparations.

\section{Endothelial Cell Culture}

Primary human coronary arterial endothelial cells (HCAEC) (Cambrex, Baltimore, MD), and pulmonary arterial endothelial cells (HPAEC) (Cambrex) were used. Data shown are from three different donors. Human umbilical vein (HUVEC) (ATCC, Manassas, VA) endothelial cells were from a single donor. All cells were cultured in Clonetics endothelial cell basal media (Cambrex) containing factors provided in the Singlequot Bullet Kit (Cambrex).

\section{Adenoviral Infection of Endothelial Cell Cultures}

Cells were seeded at 1x10E5 cells per well in 12-well plates, 16 hours prior to infection. Cells were infected with $10 \mathrm{vp} / \mathrm{cell}$ in endothelial cell basal media in a minimal volume of $0.2 \mathrm{~mL}$ per well. After $3 \mathrm{~h}$, virus-containing media was removed and fresh media was added. After $48 \mathrm{~h}$, cell culture media was removed and cells were washed one time with phosphate buffered saline. Luciferase activity was measured using the Luciferase Assay System (Promega), according to manufacturer's instructions.

\section{RESULTS AND DISCUSSION}

In this study, we focused on three types of Ad modifications utilized by our laboratory for CAR-independent infection. A schematic representation of the genetically modified Ad5 vectors studied is shown in Fig. (1).

We first evaluated capsid modifications in which short peptide motifs were incorporated into the knob domain of the Ad5 fiber protein, as shown in Fig. (1), panel B (Ad5.pK7, Ad5.RGD, Ad5.RGD.pK7). In these vectors, the incorporated pK7 motif (Ad5.pK7) allows the Ad5 to bind to heparin-sulfate containing receptors and the RGD motif (Ad5.RGD) allows the Ad5 to interact with integrins. Further, Ad5.RGD.pK7 embodies both of these modifications together to increase infectivity by potentially utilizing multiple types of receptors simultaneously. In the second type of vectors, we evaluated the infectivity of an Ad5 in which the capsid was genetically modified to include a chimeric fiber, consisting of an Ad5 tail and shaft, but the knob domain from human Ad serotype 3. This knob domain provides
CAR-independent infection (Fig. (1), panel B, Ad5/3Luc1). Finally, we evaluated the infectivity of two chimeric Ad5 viruses, in which the knob domain of fiber is replaced with that of canine Ad serotypes 1 or 2 (Ad5Luc1-CK1 and Ad5Luc1-CK2 respectively) (Fig. (1), panel B, Ad5Luc1CK1 and Ad5Luc1-CK2).

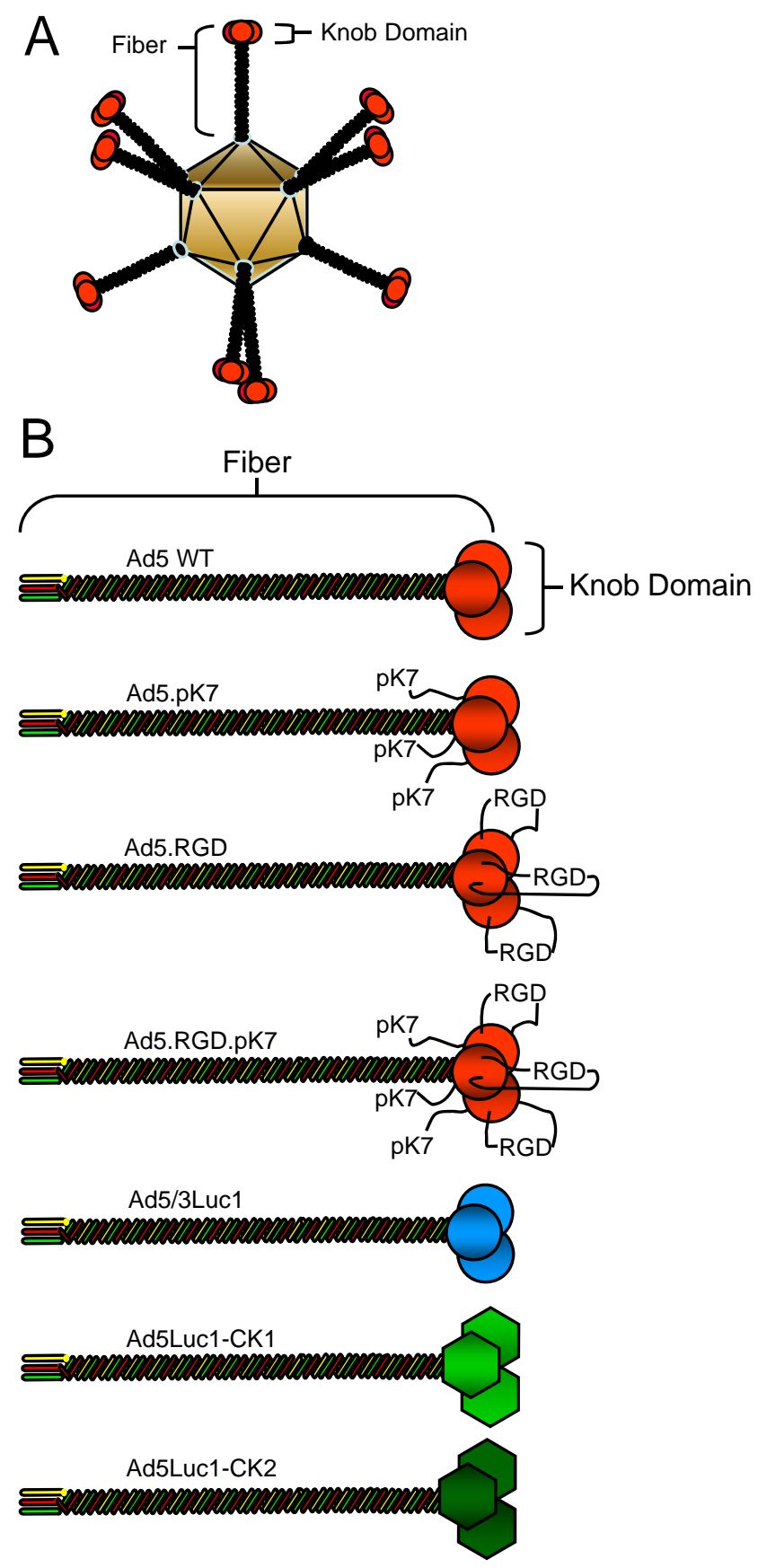

Fig. (1). Schematic representation of a generalized Ad vector (panel A), which can be genetically modified for enhanced infectivity by incorporation of peptide motifs or alternate fiber knob domains as shown in panel $\mathbf{B}$. Wild type unmodified adenovirus serotype 5 fiber and knob domain is shown first (Ad5WT), then with increasingly complex incorporated peptide motifs (Ad5.pK7, Ad5.RGD, and Ad5.RGD.pK7) followed by incorporation of serotype $3 \mathrm{knob}$ (Ad5/3Luc1) and replacement of the Ad5 knob domain with the knob domains from canine adenovirus serotypes 1 or 2 (Ad5Luc1-CK1 and Ad5Luc1-CK2 respectively). 
First, to determine the ability of our control vectors (Ad5Luc1 and Ad5GL) to infect endothelial cells, we infected HPAEC, HCAEC and HUVEC. Fig. (2) shows the ability of unmodified Ad5 to infect each primary human endothelial cell type. While the vectors used in panels A and B differ in the type of expression cassette contained, both have native Ad5 fibers and show a similar trend in gene transfer between cell types. In both panels, the HPAEC are shown to be approximately 3 -fold more susceptible to Ad5 infection compared to HCAEC. HCAEC were generally 2 -fold more susceptible to infection compared to the HUVEC. Of these cell types, the HUVEC were the most refractory to Ad5 infection as reflected by 10 -fold less luciferase activity than the HPAEC.

A

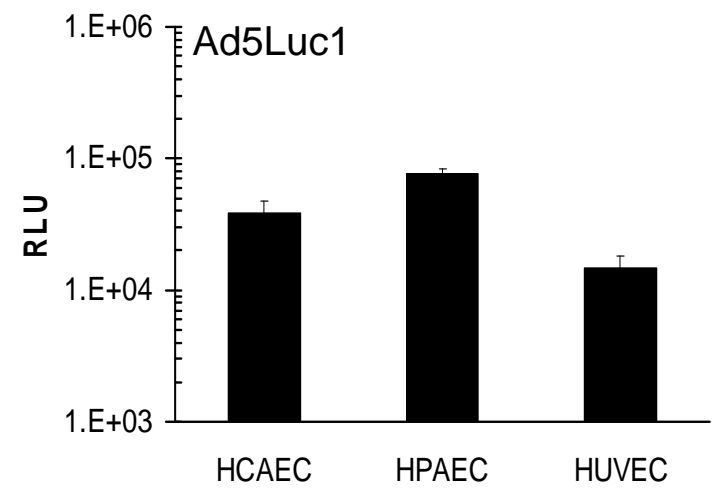

B

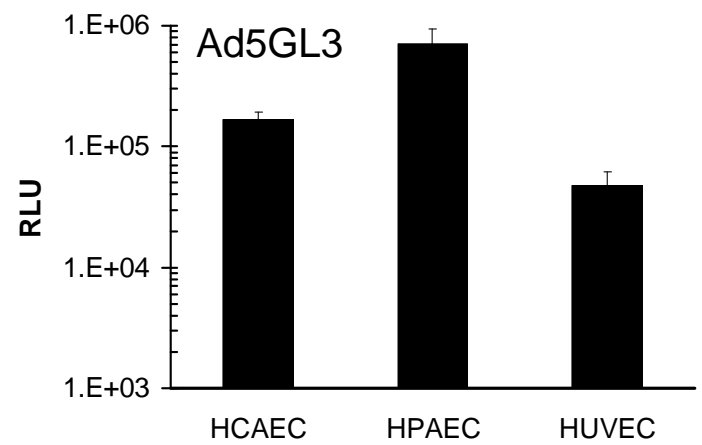

Fig. (2). Luciferase activity expressed as relative light units (RLU) following infection with Ad5 wild type capsid control virus for 48h. Ad5Luc1 contains firefly luciferase as a reporter gene (panel A) and Ad5GL3 contains a dual reporter cassette with green fluorescent protein and luciferase (panel B).

Upon establishing native Ad5 infectivity in the three cell types, we next sought to compare this to each tropismmodified virus. First, we analyzed gene transfer efficiency of our vector panel in HPAEC, which were most susceptible to Ad5 infection. In these cells, the greatest infectivity enhancement was achieved using Ad5/3Luc1, providing a 26fold increase compared to Ad5. Vectors modified to contain knobs from either canine Ad serotype 1 (Ad5Luc1-CK1) or 2 (Ad5Luc1-CK2) provided a limited increase in infectivity (4.3 and 2.4-fold respectively). Other fiber modifications such as Ad5.RGD and the mosaic Ad5.RGD.pK7 also showed limited increases in infectivity of 3.8 , and 1.1 fold respectively. Ad5.pK7 exhibited decreased infectivity by $50 \%$.
In a trend similar to HPAEC, the Ad5/3Luc1 vector demonstrated increased infectivity in HCAEC (29.6-fold, shown in panel B of Fig. (3)). In this second human primary arterial cell type, all other capsid modifications showed infectivity ranging from less than the wild type capsid (Ad5.RGD.pK7) to 6.8-fold (Ad5.RGD).

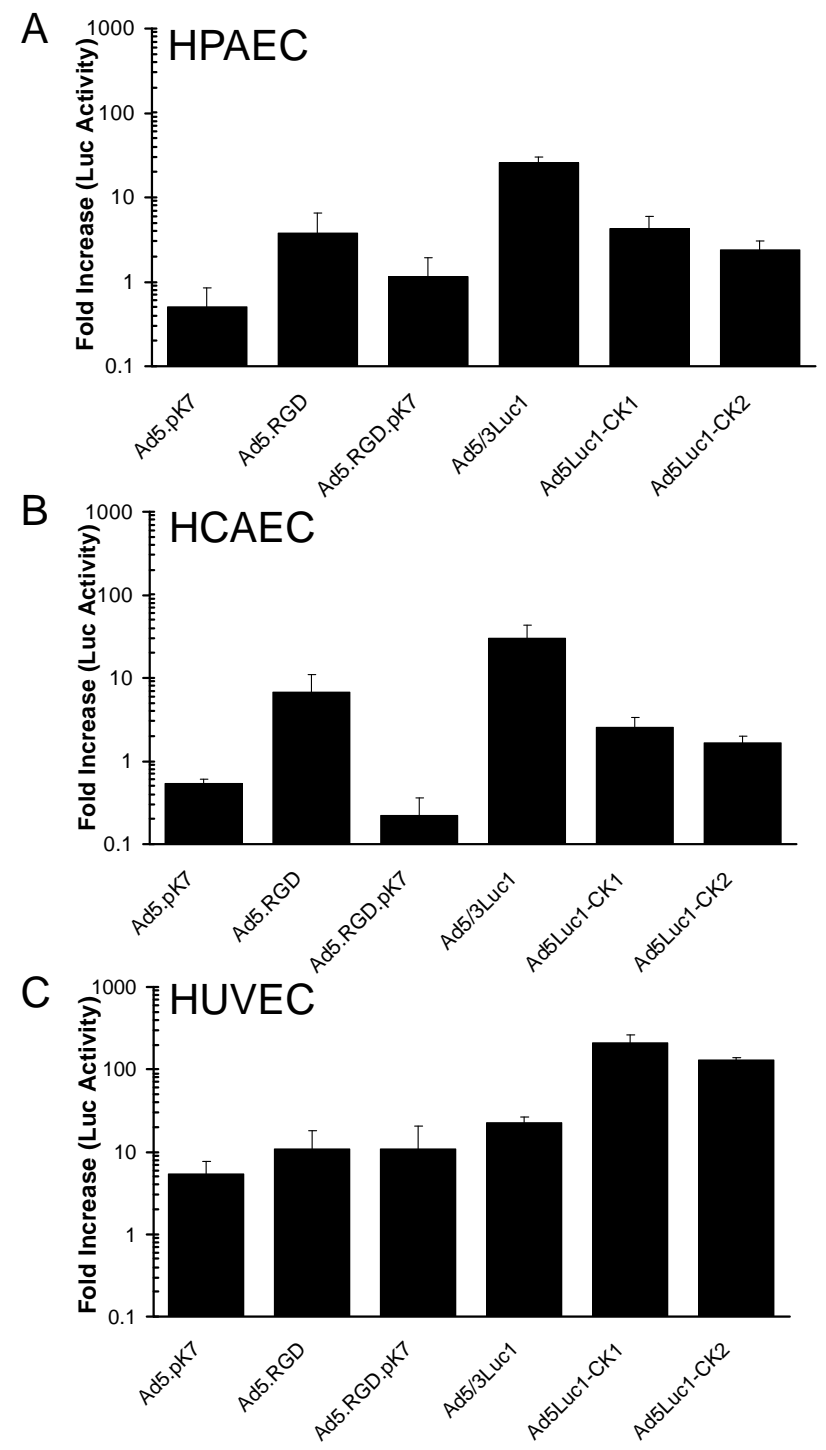

Fig. (3). Fold increase in luciferase activity versus Ad5 vectors with native fibers for a panel of genetically capsid modified Ad vectors in human pulmonary artery (HPAEC), human coronary artery (HCAEC) and human umbilical vein (HUVEC) endothelial cells. Bars indicate the mean fold increase in luciferase compared to isogenic control ( $n=3, \pm$ standard deviation).

In contrast to the primary endothelial cell types of arterial origin, HUVEC showed the largest gains in luciferase activity by the vectors modified to display canine Ad fiber knob domains. In particular, infectivity compared to Ad5 was increased 213-fold and 130-fold for Ad5Luc1-CK1 and Ad5Luc1-CK2 respectively. Similar to the other primary endothelial cells, Ad5/3Luc1 increased infectivity 22-fold. The small heterologous peptide Ads (AdpK7, AdRGD, and AdRGDpK7) increased infectivity less than 10-fold.

In this study, we present data demonstrating that tropismmodified Ad5-based vectors can increase gene delivery to 
human primary endothelial cells. Increases were accomplished via incorporation of various peptide motifs or knob domains originating from human Ad serotype 3 or canine Ads. Gains in infectivity were attained in the two arterial cell types, pulmonary and coronary in origin, via Ad5/3Luc1. This tropism-modified Ad increased infectivity more than 25-fold compared to Ad5. However, in the HUVEC, infectivity was increased greater than 100-fold using knob domains from either canine Ad serotype 1 or 2 . Taken together, these data demonstrated that gains in infectivity can be achieved by incorporating fiber knob domains from alternate human Ad serotypes and non-human xenotypes.

To explore the utility of modifying the knob domain of the Ad5 fiber protein, we first sought to explore the utility of polypeptide motifs to increase Ad5 infectivity. Previous studies have used phage display to identify motifs that were subsequently incorporated into Ad5. In these vectors, the capsid modifications were able to increase infectivity in human saphenous vein endothelial cells [21]. In the present study, we examined the utility of a polylysine (pK7) and integrin binding (RGD) motif. In addition, we investigated the creation of a mosaic virus that could potentially utilize both motifs to increase endothelial cell infection. Whereas the RGD motif increased the Ad5 infection in all three types of endothelial cells, the pK7 motif did not increase infectivity in HCAEC and HPAEC. The RGD and pK7 motifs did increase infection to a minor extent in the HUVEC. The combination of the two peptide motifs did not produce gains in infectivity in the HPAEC or HCAEC. In HUVEC, the combination of the two motifs did produce a gain in infectivity compared to Ad5. However, this increase was not greater than the use of the RGD motif alone.

The idea of using alternate human serotype fiber or knob domains to increase infectivity for Ad5 is not new. In fact, the gains in infectivity seen with Ad5/3Luc1 lend credence to many other previous studies utilizing human serotype knob domains in the Ad5 capsid. The human adenovirus serotype 3 knob domain was previously incorporated into Ad5 vector in a similar fashion to the Ad5/3Luc1 reported here. The authors reported that this vector increased infectivity in HCAEC but not in HUVEC [22]. In the current study, Ad5/3Luc1 increased infection in HCAEC as well as in HUVEC. This discrepancy in HUVEC infectivity may be due in part to the variation in cellular receptors. This phenomenon is commonly seen in HUVEC and other primary endothelial cells. For example, CAR expression in HUVEC has been shown to be influenced by many factors including cellular activation [23]. Thus, variations in culture conditions and origin of the tissue also may account for the discrepancy in HUVEC infectivity with Ad5/3Luc1 compared to the previous study. Similar to this study, other reports using Ad vectors with alternate serotype knob domains have shown gains in infectivity in endothelial cells from such tropism modification. For example, using the fiber knob domain of Ad serotype 16, Denby et al demonstrated that the incorporation of this domain into Ad5 could increase infection of human saphenous vein endothelial cells [24]. Further, Ad serotype 35 knob domain has shown to increase infection in HUVEC and HCAEC [25]. It also bears noting that adenovirus type 16 , type 3 and type 35 may also utilize the same receptor, CD46, as they are all subgroup B adenoviruses. [26]. However, in the case of serotype 3, the CD46 receptor was shown to mediate infection but use of an anti-CD46 antibody was unable to inhibit infection [27]. Together, these reports support the utility of alternate human serotype domains for infectivity gains in human endothelial cells.

Although previous reports in the literature have explored the use of alternate human serotype domains to increase infectivity in many cell types, this is the first study to show the ability of fiber knob domains from Ad viruses associated with non-human animals to increase infection in human primary endothelial cells. Whereas infection with Ad5Luc1CK1 and Ad5Luc1-CK2 increased infectivity in HUVEC 100 and 200-fold respectively, the exact mechanisms of binding and internalization of these canine Ads are not yet fully characterized. Studies have shown that canine Ad 2 knob binds to CAR but the presence of recombinant Ad5 knob only partially blocks this binding [19,28]. Also, CK1 appears to display CAR-independent infection [19]. Thus, Ad5 with canine Ad knob domains may not be completely CAR-independent. In this case, it is not surprising that these vectors showed such high gains in infectivity. It would be of interest for future studies with these vectors to compare the levels of infectivity in cells with and without CAR expression to deduce the precise role of CAR expression in canine Ad knob-mediated infection. CAR expression in HUVEC and HCAEC is reported to be relatively low and similar between the two cell types [27]. However, due to variations attributed to cell culture conditions as well as differences from one preparation to another, future studies must be carefully planned to minimize these effects.

In conclusion, we have clearly shown that the incorporation of an alternate knob domain from serotype $3 \mathrm{Ad}$ and the xenotype fiber knobs from canine Ads can produce substantial gains in infectivity in endothelial cells. These gains can be exploited for scientific discovery and potentially therapeutic applications of next generation gene therapy vectors.

\section{LIST OF ABBREVIATIONS}

$\begin{array}{lll}\text { Ad5 } & = & \text { Adenovirus serotype } 5 \\ \text { CAR } & = & \text { Coxsackie and adenovirus receptor } \\ \text { RGD } & \text { Arg-Gly-Asp motif } \\ \text { HCAEC } & =\text { Human coronary artery endothelial cells } \\ \text { HPAEC } & =\text { Human pulmonary artery endothelial cells } \\ \text { HUVEC } & =\text { Human umbilical vein endothelial cells } \\ \text { PFU } & =\text { Plaque forming units } \\ \text { RLU } & =\text { Relative light units }\end{array}$

\section{CONFLICT OF INTEREST}

The authors have no conflicts of interest.

\section{ACKNOWLEDGEMENT}

The work contained in this manuscript is supported by NIH/NHLBI \# P01HL076540.

\section{REFERENCES}

[1] Aird WC. Endothelial cell heterogeneity. Crit Care Med 2003; 31(4 Suppl): S221-30

[2] Mathis JM, Stewart PL, Zhu ZB, Curiel DT. Advanced generation adenoviral virotherapy agents embody enhanced potency based 
upon CAR-independent tropism. Clin Cancer Res 2006; 12(9): 2651-6.

[3] Henry LJ, Xia D, Wilke ME, Deisenhofer J, Gerard RD. Characterization of the knob domain of the adenovirus type 5 fiber protein expressed in Escherichia coli. J Virol 1994; 68(8): 5239-46.

[4] Xia D, Henry LJ, Gerard RD, Deisenhofer J. Crystal structure of the receptor-binding domain of adenovirus type 5 fiber protein at 1.7 A resolution. Structure 1994; 2(12): 1259-70.

[5] Tomko RP, Xu R, Philipson L. HCAR and MCAR: the human and mouse cellular receptors for subgroup $\mathrm{C}$ adenoviruses and group $\mathrm{B}$ coxsackieviruses. Proc Natl Acad Sci USA 1997; 94(7): 3352-6.

[6] Bergelson JM, Cunningham JA, Droguett G, et al. Isolation of a common receptor for Coxsackie $\mathrm{B}$ viruses and adenoviruses 2 and 5. Science 1997; 275(5304): 1320-3.

[7] Louis N, Fender P, Barge A, Kitts P, Chroboczek J. Cell-binding domain of adenovirus serotype 2 fiber. J Virol 1994; 68(6): 4104-6.

[8] Davison E, Diaz RM, Hart IR, Santis G, Marshall JF. Integrin alpha5beta1-mediated adenovirus infection is enhanced by the integrin-activating antibody TS2/16. J Virol 1997; 71(8): 6204-7.

[9] Li E, Brown SL, Stupack DG, Puente XS, Cheresh DA, Nemerow GR. Integrin alpha(v)beta1 is an adenovirus coreceptor. J Virol 2001; 75(11): 5405-9.

[10] Wickham TJ, Mathias P, Cheresh DA, Nemerow GR. Integrins alpha $\mathrm{v}$ beta 3 and alpha $\mathrm{v}$ beta 5 promote adenovirus internalization but not virus attachment. Cell 1993; 73(2): 309-19.

[11] Salone B, Martina Y, Piersanti S, et al. Integrin alpha3beta1 is an alternative cellular receptor for adenovirus serotype 5. J Virol 2003; 77(24): 13448-54

[12] Bai M, Harfe B, Freimuth P. Mutations that alter an Arg-Gly-Asp (RGD) sequence in the adenovirus type 2 penton base protein abolish its cell-rounding activity and delay virus reproduction in flat cells. J Virol 1993; 67(9): 5198-205.

[13] Glasgow JN, Everts M, Curiel DT. Transductional targeting of adenovirus vectors for gene therapy. Cancer Gene Ther 2006; 13(9): 830-44

[14] Carson SD, Hobbs JT, Tracy SM, Chapman NM. Expression of the coxsackievirus and adenovirus receptor in cultured human umbilical vein endothelial cells: regulation in response to cell density. $\mathbf{J}$ Virol 1999; 73(8): 7077-9.

[15] Raschperger E, Thyberg J, Pettersson S, Philipson L, Fuxe J, Pettersson RF. The coxsackie- and adenovirus receptor (CAR) is an in vivo marker for epithelial tight junctions, with a potential role in regulating permeability and tissue homeostasis. Exp Cell Res 2006; 312(9): 1566-80.
[16] Dmitriev I, Krasnykh V, Miller CR, et al. An adenovirus vector with genetically modified fibers demonstrates expanded tropism via utilization of a coxsackievirus and adenovirus receptorindependent cell entry mechanism. J Virol 1998; 72(12): 9706-13.

[17] Wu H, Seki T, Dmitriev I, et al. Double modification of adenovirus fiber with RGD and polylysine motifs improves coxsackievirusadenovirus receptor-independent gene transfer efficiency. Hum Gene Ther 2002; 13(13): 1647-53.

[18] Krasnykh VN, Mikheeva GV, Douglas JT, Curiel DT. Generation of recombinant adenovirus vectors with modified fibers for altering viral tropism. J Virol 1996; 70(10): 6839-46.

[19] Stoff-Khalili MA, Rivera AA, Glasgow JN, et al. A human adenoviral vector with a chimeric fiber from canine adenovirus type 1 results in novel expanded tropism for cancer gene therapy. Gene Ther 2005; 12(23): 1696-706.

[20] Glasgow JN, Kremer EJ, Hemminki A, Siegal GP, Douglas JT, Curiel DT. An adenovirus vector with a chimeric fiber derived from canine adenovirus type 2 displays novel tropism. Virology 2004; 324(1): 103-16.

[21] Nicklin SA, White SJ, Nicol CG, Von Seggern DJ, Baker AH. In vitro and in vivo characterisation of endothelial cell selective adenoviral vectors. J Gene Med 2004; 6(3): 300-8.

[22] Stevenson SC, Rollence M, Marshall-Neff J, McClelland A. Selective targeting of human cells by a chimeric adenovirus vector containing a modified fiber protein. J Virol 1997; 71(6): 4782-90.

[23] Jornot L, Morris MA, Moix I, Rochat T. N-acetylcyteine augments adenovirus-mediated gene expression in human endothelial cells by enhancing transgene transcription and virus entry. J Gene Med 2002; 4: 54-65.

[24] Denby L, Work LM, Graham D, et al. Adenoviral serotype 5 vectors pseudotyped with fibers from subgroup D show modified tropism in vitro and in vivo. Hum Gene Ther 2004; 15(11): 1054-64.

[25] Shinozaki K, Suominen E, Carrick F, et al. Efficient infection of tumor endothelial cells by a capsid-modified adenovirus. Gene Ther 2006; 13(1): 52-9.

[26] Fleischli C, Sirena D, Lesage G, et al. Species B adenovirus serotypes $3,7,11$, and 35 share similar binding sites on the membrane cofactor protein CD46 receptor. J Gen Virol 2007; 88(pt11): 2925 34.

[27] Marttila M, Persson D, Gustafsson D, et al. CD46 is a cellular receptor for all species $\mathrm{B}$ adenoviruses except types 3 and 7 . J Virol 2005; 79(22): 14429-36.

[28] Kremer EJ. CAR chasing: canine adenovirus vectors-all bite and no bark? J Gene Med 2004; 6 (Suppl 1): S139-51. 